\title{
1 Chemical Modification of Nanocellulose with Canola Oil Fatty Acid Methyl Ester
}

2 Liqing Wei, ${ }^{a}$ Umesh P. Agarwal, ${ }^{a}$ Kolby C. Hirth, ${ }^{a}$ Laurent M. Matuana, ${ }^{b}$ Ronald C. Sabo, ${ }^{\text {a* }}$

3 Nicole M. Stark $\mathrm{a}^{\mathrm{a}^{*}}$

$4{ }^{\mathrm{a} U S D A}$ Forest Service, Forest Products Laboratory, One Gifford Pinchot Drive, Madison, WI $5 \quad$ 53726, United States

$6 \quad{ }^{\mathrm{b}}$ School of Packaging, Michigan State University, East Lansing, MI 48824, USA

7 Corresponding authors

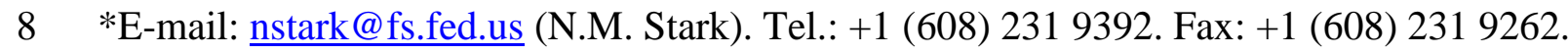

9 *E-mail: rsabo@fs.fed.us (R.C. Sabo). Tel.: +1 (608) 231 9530. Fax: +1 (608) 2319262.

10 Abstract: Cellulose nanocrystals (CNCs), produced from dissolving wood pulp, were

11 chemically functionalized by transesterification with canola oil fatty acid methyl ester (CME).

12 CME performs as both the reaction reagent and solvent. Transesterified CNC (CNCFE) was

13 characterized for their chemical structure, morphology, crystalline structure, thermal stability,

14 and hydrophobicity. Analysis by Fourier transform infrared (FTIR) and FT-Raman

15 spectroscopies showed that the long chain hydrocarbon structure was successfully grafted onto

16 CNC surfaces. After transesterification the crystal size and crystallinity of nanocrystals were not

17 changed as determined by Raman spectroscopy and wide angle X-ray diffraction (XRD).

18 CNCFE showed higher thermal stability and smaller particle size than unmodified CNCs. Water

19 contact angle measurement indicated the CNCFE surface has significantly higher hydrophobicity

20 than unmodified CNCs. The transesterified CNCs could be potentially used as hydrophobic

21 coatings and reinforcing agents to hydrophobic polymer for nanocomposites. 
22 Keywords: Nanocellulose; transesterification; crystallinity; vegetable oil; hydrophobiciy

\section{1. Introduction}

Biobased materials are attracting immense research interest because of their low negative

26 impact to environment at the end of service life. Cellulose nanomaterials have been known more

27 than half century ago and have gained extraordinarily renewed attention in recent years.

28 Generally, two forms of nanocellulose, the cellulose nanocrystals ( $\mathrm{CNCs})$ and cellulose

29 nanofibrils (CNF), have been produced. CNCs exhibit impressive properties such as low-density,

30 high mechanical strength, low thermal expansion, and excellent biocompatibility and

31 renewability (Moon, Beck, \& Rudie, 2013). The tensile modulus of CNCs can reach to $170 \mathrm{GPa}$

32 because of its high crystallinity (54-90\%) (Habibi, Lucia, \& Rojas, 2010; Klemm et al., 2011;

33 Wei \& McDonald, 2016). This has shown great promise of CNCs as reinforcing agents (Bardet,

34 Roussel, Coindeau, Belgacem, \& Bras, 2015; Habibi et al., 2010; Moon et al., 2013; Pereira et al.,

35 2014). CNCs can be produced from cellulose derived from trees, plants, tunicates, bacteria and

36 algae. Acid hydrolysis, with sulfuric acid generally, is used to remove most amorphous fractions

37 of cellulose to prepare CNCs (Moon et al., 2013; Reiner \& Rudie, 2013). The negatively charged

38 surfaces of CNCs result in stable colloidal suspensions of CNC in water. However, similar to

39 general cellulose, the abundant hydroxyl groups on CNC surfaces make it a highly hydrophilic

40 material, which often impairs its excellent properties.

41 In this situation, various approaches have been investigated to modify $\mathrm{CNC}$ and tune its

42 surface chemistry. In some methods, CNCs are modified during the production step (Boujemaoui,

43 Mongkhontreerat, Malmström, \& Carlmark, 2015; Espino-Pérez, Domenek, Belgacem, Sillard, 
44 \& Bras, 2014), while in other strategies the CNCs were produced first followed by the

45 modifications, such as acetylation (Abraham et al., 2016; Lin, Huang, Chang, Feng, \& Yu, 2011;

46 Ramírez, Fortunati, Kenny, Torre, \& Foresti, 2016) and polymer grafting (Eyley \& Thielemans,

47 2011; Hatton, Kedzior, Cranston, \& Carlmark, 2017; Huang et al., 2016; Morandi, Heath, \&

48 Thielemans, 2009). The main challenge with chemical functionalization to CNCs is to select an

49 appropriate method, namely the surface modification that would not impact the distinctive

50 properties of CNCs. For example, if the 3D crystal network is interrupted during modification

51 the mechanical properties of CNCs could deteriorate, which could consequently limit the

52 applications of modified CNC (Klemm, Heublein, Fink, \& Bohn, 2005; Klemm et al., 2011).

53 Those mentioned CNC modification approaches can be relatively easily achieved with the use of

54 organic solvents or toxic reagents, such as dimethylformamide, toluene diisocyanate, and

55 halogenated solvent. In addition, some water based approaches have been used to modify CNC

56 surfaces, for example, Zaman and coworkers have used glycidyltrimethylammonium chloride to

57 modify $\mathrm{CNC}$ to render its cationic surfaces formed during acid hydrolysis process (Zaman, Xiao,

58 Chibante, \& Ni, 2012). Yoo and Youngblood have applied an one-pot synthesis approach to graft

59 lactic acid onto CNC surfaces to produce a CNC-grafted-PLA copolymer (Yoo \& Youngblood,

60 2016). However, the hydrophobicity of modified CNCs was not increased. Thus, the preparation

61 of modified CNCs with intact properties by using "sustainable chemistry" based strategy is still

62 challenging in nanocellulose research.

63 Vegetable oil, a triglyceride, is a promising renewable resource and after transesterification

64 reaction with an alcohol (commonly methanol) the fatty acid methyl ester (FAME) is produced

65 (Lang, Dalai, Bakhshi, Reaney, \& Hertz, 2001). Most common fatty acids present in vegetable

66 oils include palmitic (C16:0), stearic (C18:0), oleic (C18:1), linoleic (C18:2), linolenic (C18:3), 
67 and ricinoleic $(\mathrm{C} 18: 1, \mathrm{OH})$ acids. While the long hydrocarbon chain of the fatty acid continues to

68 be strongly hydrophobic, the presence of the carboxylic acid group at one end of the molecule

69 adds some hydrophilic properties to fatty acids (Yoo \& Youngblood, 2016). When fatty acids are

70 converted into FAME, the hydrophobic long chain hydrocarbon structure could be chemically

71 bonded to $\mathrm{CNCs}$ by the second transesterification reaction between the hydroxyl $(\mathrm{OH})$ groups

72 and FAME. To our knowledge, this type of transesterification has not been studied for the

73 modification of nanocellulose.

74 In this study, CNCs were produced by sulfuric acid hydrolysis of wood-derived dissolving

75 pulp followed by freeze-dried process. FAME was prepared by the first transesterification

76 reaction between canola oil and methanol, and then used to modify CNCs via the second

77 transesterification. GC-MS was used to determine the molecular weight of FAME adducts. The

78 chemical and crystalline structures of produced transesterified CNCs were characterized by FT-

79 IR, FT-Raman and XRD analyses. Thermal stability was studied by thermogravimetric analysis

80 (TGA). Surface hydrophobicity of CNCs was evaluated by water contact angle measurement.

81 The results provide insight into an environmentally benign chemical modification of CNC to

82 broaden its application as hydrophobic coatings and reinforcing agent for hydrophobic polymers.

84 2. Experimental

85 2.1. Materials

86 Cellulose nanocrystals (CNCs, 2014-FPL-CNC-065) were produced by USDA-Forest

87 Service, Forest Products Laboratory (Madison, Wisconsin) using sulfuric acid hydrolysis method.

88 Briefly, the prehydrolyzed kraft rayon-grade dissolving Eucalyptus dry lap wood pulp was used 
89 as the starting material for producing the CNCs. The dissolving wood pulp contains $78.1 \%$,

$9015.5 \%$, and $0.1 \%$ of cellulose, hemicellulose, and Klason lignin, respectively (Wang et al., 2012).

91 Bundles of the dissolving pulp were reacted with $64 \%$ sulfuric acid (acid:cellulose $=8: 1(\mathrm{v}: \mathrm{w}))$

92 at $45^{\circ} \mathrm{C}$ for about $1.5 \mathrm{~h}$ under a nitrogen blanket with constant stirring. The hydrolysis was

93 quenched by diluting the reaction mixture with distilled water to approximately a 10-fold volume

94 of the initial mixture. The $\mathrm{CNC}$ were neutralized by adding a $\mathrm{NaOH}$ solution $(5 \mathrm{wt} \%)$. The

95 sodium sulfate and other salts were removed by ultrafiltration to retain the CNC concentration at

96 approximately $1 \%$. After all salts are removed, the CNC suspension was concentrated to have

$9710.7 \mathrm{wt} \%$ of solid (Reiner \& Rudie, 2013). The liquid CNC suspension (10.7 wt \%) was freeze-

98 dried to obtain dry CNCs materials. The sulfur content of CNCs was analyzed using an ICP-AES

99 (Ultima II, Horiba Jobin-Yvon, Edison, NJ, USA) to indicate the cellulose surface sulfation or

100 charge (Chen et al., 2015), and the final CNCs have $0.94 \mathrm{wt} \%$ sulfur in sodium form.

101 ACS grade $\mathrm{NaOH}(99.5 \%), \mathrm{KOH}(99.5 \%), \mathrm{K}_{2} \mathrm{CO}_{3}(99 \%)$, hexane, and methanol (MeOH, $10299.8 \%$ ) were all purchased from Fisher Scientific Co. (Pittsburgh, PA, USA). All chemicals 103 were directly used as received without purification.

104 2.2. Preparation of canola oil fatty acid methyl ester (CME)

105 Commercial canola oil (sold under the name "Nature's Secret") was purchased from a local

106 Costco warehouse (Middleton, WI). Canola oil fatty acid methyl ester of (CME) was synthesized

107 by a modified method described elsewhere (Lang et al., 2001). The reaction was conducted

108 under excess methanol $(\mathrm{MeOH})$ with molar ratio of $\mathrm{MeOH}$ to oil of 6:1. Catalyst $(\mathrm{NaOH})$

109 concentration was $1 \mathrm{wt} \%$ of the oil and methanol mixture. The $\mathrm{NaOH}$ pellets were placed in

$110 \mathrm{MeOH}$ and stirred with magnetic stirrer bar until completely dissolved. This mixture (66 g) was 
111 quickly added to oil $(150 \mathrm{~g})$ in a round bottom flask $(500 \mathrm{~mL})$ and stirred for $2 \mathrm{~h}$ at $70{ }^{\circ} \mathrm{C}$. The

112 reaction was conducted using a reflux apparatus with a condenser was used. After the reaction

113 was complete, crude glycerol was separated in a separatory funnel. The top ester layer was

114 dissolved in $\mathrm{KOH}(0.5 \mathrm{wt} \%$ of the mixture) solution of $\mathrm{MeOH}$ for the second reaction for $2 \mathrm{~h}$ at

$11525{ }^{\circ} \mathrm{C}$ to ensure full conversion to $\mathrm{CME}$. The crude CME was separated and washed with hot

116 water $\left(70^{\circ} \mathrm{C}\right)$ until the liquid was transparent and neutral $\mathrm{pH}$. Traces of non-reacted $\mathrm{MeOH}$ were

117 removed by purging with $\mathrm{N}_{2}$ for $12 \mathrm{~h}$.

\section{2.3. Canola oil fatty acid methyl ester composition by GC-MS}

119 The CME (2.0 mg) was weighed into a $\mathrm{GC}$ vial to which $2 \mathrm{~mL}$ of $\mathrm{CH}_{2} \mathrm{Cl}_{2}$ was added.

$120 \mathrm{CH}_{2} \mathrm{Cl}_{2}$ contained 1-napthalaneacetic acid methyl ester as an internal standard $(50 \mu \mathrm{g} / \mathrm{mL})$. The

121 as-prepared samples were analyzed using a Varian (Walnut Creek, CA, USA) model 3800 gas

122 chromatograph interfaced to a Varian model 4000 ion trap mass detector, operated in full scan EI

123 mode at $70 \mathrm{eV}$ in the range $\mathrm{m} / \mathrm{z}=80-600$. The transfer line was maintained at $250{ }^{\circ} \mathrm{C}$. A Restek

124 (Bellefonte, PA) Rxi-1MS column (30 m, $0.25 \mathrm{~mm}$ ID, $0.25 \mu \mathrm{m}$ thickness) was temperature

125 programmed $40{ }^{\circ} \mathrm{C}$ for $1 \mathrm{~min}$, and then ramped at $5{ }^{\circ} \mathrm{C} / \mathrm{min}$ to $290{ }^{\circ} \mathrm{C}$ for 2 mins. A CombiPal

126 auto-sampler (CTC Analytics, Zwingen, Switerzerland) equipped with a $10 \mu \mathrm{L}$ liquid syringe

127 was used for $1 \mu \mathrm{L}$ injections made in split mode 50:1, with injection port temperature of $200^{\circ} \mathrm{C}$.

128 Helium $(99.9999 \%)$ was used as carrier gas at a flow of $1 \mathrm{~mL} / \mathrm{min}$.

\section{2.4. Transesterification of CNCs}

130 Catalyst $\left(\mathrm{K}_{2} \mathrm{CO}_{3}, 0.2 \mathrm{wt} \%\right.$ of the total dry weight of CME and CNCs) was dissolved in

$131 \mathrm{MeOH}$ first with constant stirring for 30min, after which the excessive amount of CME (220 g)

132 and $\mathrm{CNC}(10 \mathrm{~g})$ powders were added (Note: the non-reacted CME can be recycled and reused to 
133 avoid waste). The reactant mixture was heated to $90{ }^{\circ} \mathrm{C}$ to drive off the $\mathrm{MeOH}$ to form a foaming 134 soap. The remaining $\mathrm{MeOH}$ was further removed by applying intermittent vacuum to the flask

135 until foaming subsided. A condenser installed to the flask and vacuum (10 mbar) applied and the 136 mixture was heated to temperature $\left(T_{R}=100\right.$ and $\left.120^{\circ} \mathrm{C}\right)$ for a defined period of reaction time $\left(t_{R}\right.$ $137=4,8,14,20$, and $30 \mathrm{~h}$ ). The CNC fatty acid ester (CNCFE) was recovered by filtration then 138 Soxhlet extracted with hexane for $12 \mathrm{~h}$ to remove non-reacted CME. The CNCFE was dried 139 under vacuum overnight and weighed to determine the weight percentage gain (WPG\%).

$140 \quad \mathrm{WPG} \%=\left(\mathrm{W}_{\mathrm{CNCFE}}-\mathrm{W}_{\mathrm{CNC}}\right) / \mathrm{W}_{\mathrm{CNC}} \times 100$

141 where $\mathrm{W}_{\mathrm{CNCFE}}$ is the dry weight of hexane extracted CNCFE after transesterification, while

$142 \mathrm{~W}_{\mathrm{CNC}}$ is the weight of CNC before the reaction. The reaction time $\left(t_{R}\right)$ and temperature $\left(\mathrm{T}_{\mathrm{R}}\right)$ were 143 attempted to tune the WPG\%. The reaction mechanism is shown in Fig. 1.

144 The number of $\mathrm{OH}$ groups substituted in $\mathrm{CNCs}$ after transesterification were estimated 145 based on WPG and the molecular weight of the adduct with an assumption of 1:1 molar reaction 146 between CME and CNC hydroxyl groups (Wei, McDonald, Freitag, \& Morrell, 2013). The 147 equation used to account for the hydrogen atom transferred from $\mathrm{CNC}$ to $\mathrm{MeOH}$ (1 in the 148 equation) is shown as follows:

$149 \mathrm{OH}$ substituted $(\mathrm{mmol} / \mathrm{g}$ dry $\mathrm{CNCs})=0.01 \times \mathrm{WPG} /($ Molecular weight of adduct -1$) \times 1000$

150 where the molecular weight of adduct was estimated from gas chromatography mass

151 spectrometry (GC-MS) analysis of CME samples. 


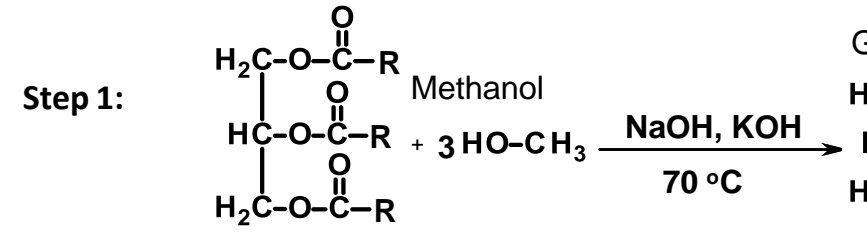

Triglycerides

(Triester of glycerol)
Glycerol

$\mathrm{H}_{2} \mathrm{C}-\mathrm{OH}$

$\mathrm{HC}-\mathrm{OH}$
$\mathrm{H}_{2} \mathrm{C}-\mathrm{OH}$

Canola oil fatty acid methyl ester (CME)

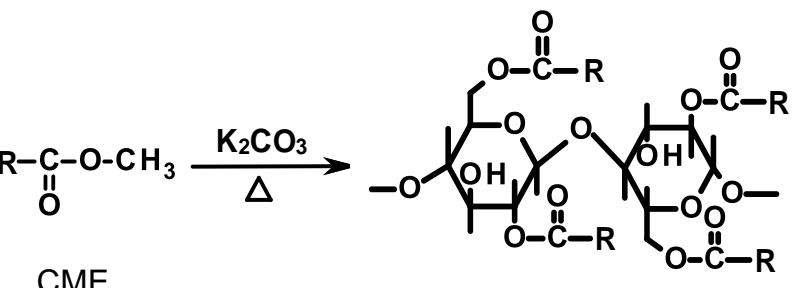

CNC fatty acid methyl ester (CNCFE)

$R$ is long chain hydrocarbons

Fig. 1 Reaction mechanism illustration for the synthesis of CME (Step 1) and CNCFE (Step 2).

\subsection{Surface chemistry of CNCs by FT-IR and FT-Raman spectroscopes}

155 Thermo Nicolet iZ10 FTIR spectrometer (attenuated total reflection (ATR) probe, Thermo

156 Scientific, Verona, Wisconsin) using a smart iTR Basic accessory. A diamond crystal with $45^{\circ}$

157 incident angle was used. The absorbance spectra were taken for an average of 128 scans in the

158 range of 4000 to $600 \mathrm{~cm}^{-1}$ with the resolution of $4 \mathrm{~cm}^{-1}$. Samples (in triplicates) were analyzed

159 after vacuum drying. The spectra were baseline corrected, averaged, and normalized to the band 160 at $1031 \mathrm{~cm}^{-1}$ using software Omnic v9.0 (Thermo Scientific).

161 Chemical and crystalline structures of unmodified CNC and CNCFE samples were analyzed

162 by Raman spectroscopy with a Bruker MultiRam spectrometer (Bruker Instruments Inc.,

163 Billerica, Massachusetts) equipped with a $1064 \mathrm{~nm} 1000 \mathrm{~mW}$ continuous-wave (CW) diode

164 pumped Nd:YAG laser. Approximately $200 \mathrm{mg}$ of each sample was pressed into a pellet. The 
165 laser power used for sample excitation was $600 \mathrm{~mW}$ per sample spectrum and 1024 scans were

166 accumulated. The Raman spectra were processed by the Bruker's OPUS software and

167 normalized to the band at $1096 \mathrm{~cm}^{-1}$.

\subsection{Thermostability of nanocrystals}

Thermogravimetric analysis (TGA) of CME, unmodified CNC, and CNCFE was conducted

170 on a PerkinElmer Pyris 1 analyzer at a heating rate of $20{ }^{\circ} \mathrm{C} /$ min under $\mathrm{N}_{2}$ atmosphere (20

$171 \mathrm{~mL} / \mathrm{min})$.

\section{2.7. Morphology}

Transmission electron microscope (TEM) was used to study the morphology of unmodified

$174 \mathrm{CNCs}$ before freeze-drying process. CNC suspension was diluted with distilled water and 175 sonicated to disperse the CNC particles, and then an aliquot was deposited on a glow-discharged

176 copper grid with formvar and carbon film (400 mesh). The grid was floated on drops of

177 approximately $5 \mu \mathrm{L}$ sample for 2 mins, and then rinsed with two consecutive $250 \mu \mathrm{L}$ drops of $2 \%$

178 aqueous uranyl acetate. Excess stain was removed by capillary action by gentle blotting. The

179 samples were imaged using a Philips CM100 TEM (Philips/FEI, Eindhoven, The Netherlands)

180 operated at $100 \mathrm{kV}$, spot $3200 \mu \mathrm{m}$ condenser aperture, and $70 \mu \mathrm{m}$ objective aperture. The images

181 were captured and recorded using a SIA L3C 4-2 M pixel CCD camera (Scientific Instruments

182 and Application, Duluth, GA).

183 Freeze-dried unmodified CNCs and transesterified CNCs power were directly deposited

184 onto carbon tape. All samples were coated with a thin layer of gold. The prepared samples were

185 investigated using a LEO Gemini field emission scanning electron microscope (SEM) operated 186 at $3 \mathrm{kV}$ under high vacuum. 


\subsection{Crystalline structure by wide angle X-ray diffraction (XRD) and FT-Raman}

The crystalline structures of unmodified CNCs and CNCFE were characterized by wide angle X-ray diffraction spectroscopy (D8 Discover diffractometer, Bruker AXS Inc., WI, USA).

190 The instrument was set up with a rotating $\mathrm{Cu} \mathrm{K} \alpha 2 \mathrm{X}$-ray IMS tube operating at $50 \mathrm{kV}$ and 1000

$191 \mu \mathrm{A}$. Scanning was performed over $2 \theta$ ranging from 5 to $60^{\circ}$ with steps of $0.005^{\circ}$. The collected

192 diffractograms were processed and peaks of interest were fitted (Gaussian function) using IGOR

193 Pro v6 software. The intensity of each peak identified by peak fitting was mathematically

194 computed. The approaches to calculate the crystallinity index of nanocrystals (CNC and CNCFE)

195 and PLA are given as follows (Segal, Creely, Martin Jr., \& Conrad, 1959):

$196 \mathrm{CrI} \%_{\mathrm{CNC} / \mathrm{CNCFE}}=\left(1-\left(\mathrm{I}_{\mathrm{am}} / \mathrm{I}_{002}\right)\right) \times 100$

197 where $I_{a m}$ is the intensity of amorphous diffraction, which is taken at $2 \theta$ angle between (002) and

198 (101) peaks where the intensity is at minimum, and $\mathrm{I}_{002}$ is the maximum intensity of the (002)

199 plane diffraction.

200 The crystal size dimension $\left(D_{\text {hkl }}\right)$ was estimated by Scherrer's formula:(Alexander, 1969)

$201 \quad \mathrm{D}_{\mathrm{hkl}}=K \times \lambda /\left(\beta_{1 / 2} \times \cos \theta\right)$

202 where $K$ is the crystal shape constant taken to be $0.9, \lambda$ is the X-ray wavelength $(\lambda=0.1542 \mathrm{~nm})$,

$203 \beta_{1 / 2}$ is the angular full width half maximum (FWHM) of the interested peak in radians obtained

204 by IGOR Pro software when peak fitting was conducted with the Gaussian function, and $\theta$ is the 205 diffraction angle in radians.

206 The spacing between the (002) planes, $d$, was calculate using the Bragg's equation:

$207 n \lambda=2 d \sin \theta$ 
208 where $n$ is an integer, $\lambda$ is the wavelength of the incident wave, and $\theta$ is the angle between the

209 incident ray and the scattering planes.

210 Crystallinities by FT-Raman were estimated using the 380-Raman method (Agarwal, Ralph,

211 \& Reiner, 2013; Agarwal, Reiner, \& Ralph, 2010) and are based on the following equations.

$212 \quad \mathrm{X}_{\text {MultiRam }}=\left(\left(\mathrm{I}_{380} / \mathrm{I}_{1,096}\right)-0.0286\right) / 0.0065$

$213 \quad \mathrm{X}_{\mathrm{RFS}-100}=\left(\mathrm{X}_{\text {MultiRam }}+2.0212\right) / 0.8222$

214 where $\mathrm{I}_{380}$ and $\mathrm{I}_{1096}$ stands for the intensity of peaks at $380 \mathrm{~cm}^{-1}$ and $1096 \mathrm{~cm}^{-1}$ for Raman spectra

215 collected by the Raman instrument, MultiRam, while $\mathrm{X}_{\mathrm{RFS}-100}$ and $\mathrm{X}_{\text {MultiRam }}$ represent the

216 crystallinity values obtained from Raman spectra collected by using two Raman instruments, the

217 RFS-100 and MultiRam, respectively.

\section{2.9. Water contact angle measurements}

219 The surface hydrophilicity of unmodified and transesterified CNCs was evaluated by contact 220 angle measurements using a KSV Attension Theta Lite Optical Tensiometer. CNC/CNCFE

221 powder was compression molded into discs $(12 \mathrm{~mm} \emptyset \times 1.5 \mathrm{~mm})$ at $20 \mathrm{MPa}$ using a hydraulic

222 press (Carver). The static contact angle between a water droplet $(10 \mu \mathrm{L})$ and the $\mathrm{CNC}$ disc was

223 measured. The contact angle was recorded from the captured images at from 0 to 10 seconds. 
A total of five different fatty acids were identified in CME, as shown in Table 1. The

228 dominant fatty acid in canola oil was oleic acid (C18:1) at $66.6 \%$, followed by linoleic acid

229 (C18:2) (23.8\%), linolenic acid (C18:3) (6.8\%), palmitic acid (C16:0) (1.8\%), and stearic acid

230 (C18:0) (1.0\%). This result agrees well to previous reports (Lang et al., 2001). It is worth noting

231 that the chemical nature of CME obtained in this study is FAME which is the constituent of

232 biodiesel. Hence, the CME to be used to modify CNCs, which will be discussed later, can be

233 substituted by commercial biodiesel with higher purity. The approximate $\mathrm{M}_{\mathrm{w}}$ of the CME

234 mixture is $294.5 \mathrm{~g} / \mathrm{mol}$, which is close to the value of $297.1 \mathrm{~g} / \mathrm{mol}$ that was reported elsewhere

235 (He, Singh, \& Thompson, 2005).

236 Table 1. Chemical composition profiles of CME determined by GC-MS.

\begin{tabular}{llll}
\hline Compounds & Retention time $(\mathrm{min})$ & $\mathrm{M}^{+}(\mathrm{m} / \mathrm{z})$ & Relative concentration $(\%)$ \\
\hline Methyl palmitate (C16:0) & 10.59 & 270 & 1.8 \\
Methyl linolenate (C18:3) & 21.47 & 292 & 6.8 \\
Methyl linoleoate (C18:2) & 21.11 & 294 & 23.8 \\
Methyl oleioate (C18:1) & 21.93 & 296 & 66.6 \\
Methyl stearate (C18:0) & 24.65 & 298 & 1.0 \\
\hline
\end{tabular}

237

238 3.2. Chemical structure changes of nanocrystals

239 In order to maximize the conversion of CNC into CNCFE via transesterification (Fig. 1),

240 excess amount of CME was used in the reaction (molar ratio of CNCs to CME = 1:12). The

241 WPG\% and the visual appearance of modified CNCs are dependent on the reaction conditions,

242 reaction time $\left(t_{R}\right)$ and reaction temperature $\left(T_{R}\right)$ (Supplementary Table $\left.S 1\right)$. When the same $T_{R}$

$243\left(100{ }^{\circ} \mathrm{C}\right)$ was used the WPG\% was increased from 5.5 to $10.0 \%$ and $10.5 \%$ with $\mathrm{t}_{\mathrm{R}}$ was

244 increased from 4 to 14 and 20 h, respectively. The WPG\% was not increased any more with the

$245 t_{R}$ was increased from 20 to $30 \mathrm{~h}$. Although higher $\mathrm{T}_{\mathrm{R}}$ yields higher WPG\%, the CNCFE showed 
246 discoloration/darkening when the reaction temperature was increased from 100 to $120{ }^{\circ} \mathrm{C}$. This

247 may be contributed to the degradation of sulfate groups on the CNCs, and relatively low thermal

248 stability is a disadvantage of CNCs produced from conventional concentrated sulfuric acid

249 hydrolysis (Chen, Zhu, Baez, Kitin, \& Elder, 2016). By changing the $t_{R}$ and $T_{R}$, WPG\% and the

$250 \mathrm{OH}$ groups substituted as well can be monitored. For example, when the $\mathrm{t}_{\mathrm{R}}$ was optimized to be

$25120 \mathrm{~h}$ at $\mathrm{T}_{\mathrm{R}}=120^{\circ} \mathrm{C}$, giving an average WPG\% (two batches) of transesterification was

$25212.5 \pm 1.2 \%$. The degree of $\mathrm{OH}$ groups substituted after transesterification was calculated from

253 equation (2) based on WPG was $0.5 \mathrm{mmol} / \mathrm{g}$ dry CNC. CNCFE sample obtained under this

254 reaction conditions was selected for further characterization. According to the final applications

255 of the transesterified $\mathrm{CNCs}$, by changing the reaction time and temperature the modification

256 efficiency (OH substitution) and appearance can be tuned.

FT-IR spectra showed that esterification induced a number of chemical changes in the CNC

258 (Fig. 2). Carbonyl groups were assigned to the aliphatic esters for CNCFE at $1730 \mathrm{~cm}^{-1}$ (Wei et

259 al., 2013). This provides evidence that transesterification was successful. The degree of

260 transesterification $\left(D_{1}\right)$ was estimated from the ratio of $\mathrm{I}_{1730} / \mathrm{I}_{1031}$ to be 0.1 , where $\mathrm{I}_{1730}$ and $\mathrm{I}_{1031}$

261 are the intensity of the $\mathrm{C}=\mathrm{O}$ stretching band and the $\mathrm{C}-\mathrm{O}$ stretching of cellulose backbone at

262 about $1730 \mathrm{~cm}^{-1}$ and $1031 \mathrm{~cm}^{-1}$, respectively. It is worth noting that the $\mathrm{D}_{1}$ value was lower than

263 the degree of acetylation of CNC by vinyl acetate reported by Ramírez et al., which used citric

264 acid as catalyst (Ramírez et al., 2016). The band for CNCs at $1640 \mathrm{~cm}^{-1}$ is ascribed to the

265 absorbed water (H-O-H) which was absent in CNCFE (Jabbari, Wisniewski, \& Peppas, 1993). A

266 new band at $1590 \mathrm{~cm}^{-1}$ was observed in the spectrum of $\mathrm{CNCFE}$ which is assigned to $\mathrm{C}=\mathrm{C}$

267 stretching within the hydrocarbon chain of unsaturated fatty acid fragments such as C18:1, C18:2,

268 and C18:3 (Mayo, Miller, \& Hannah, 2003). In addition, no peak (or even a shoulder) was 
269 observed at $2853 \mathrm{~cm}^{-1}\left(-\mathrm{O}-\mathrm{CH}_{3}\right.$ stretch), indicating the CME was removed completely by hexane 270 extraction. This further supported that the transesterification was successful.

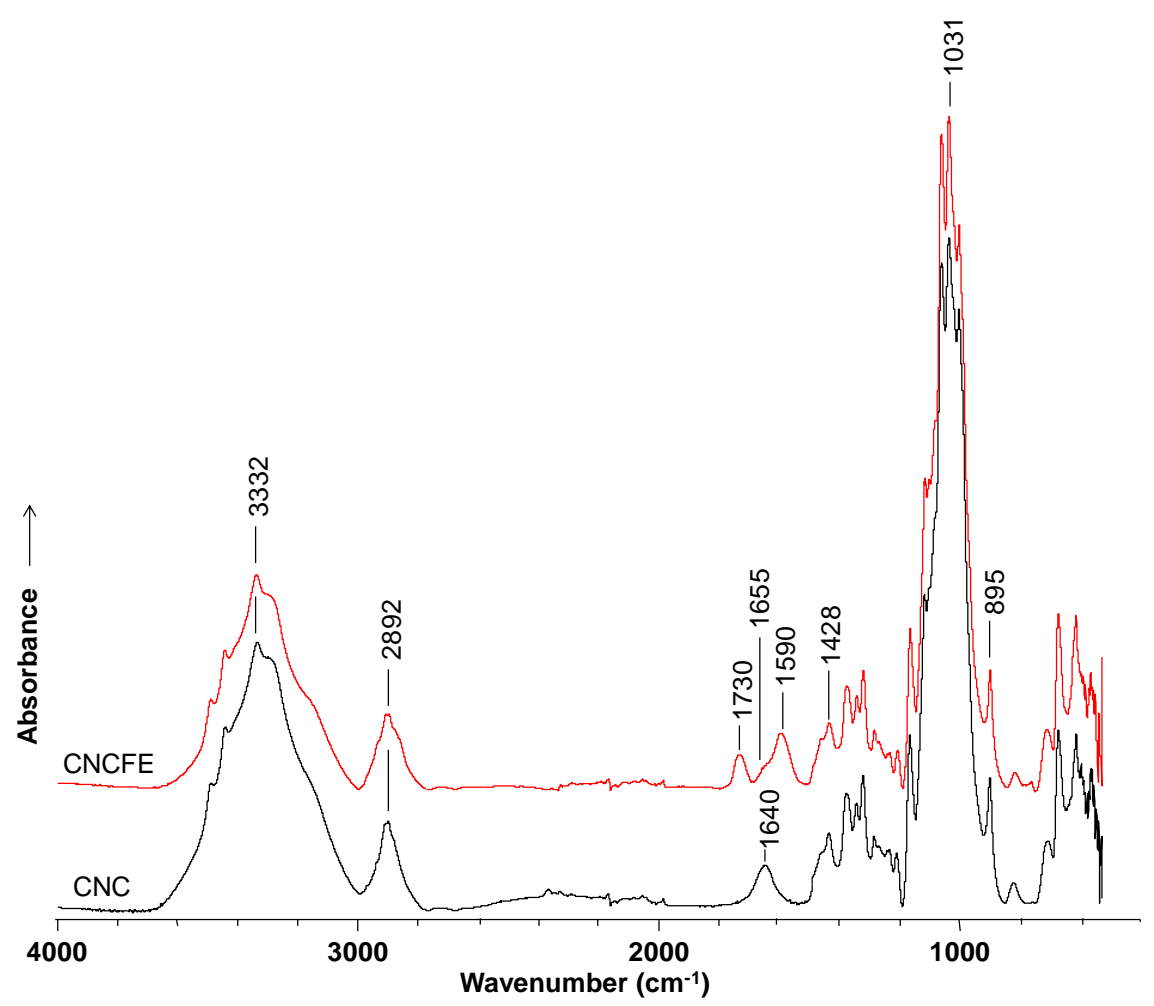

Fig. 2 FT-IR spectra of unmodified and transesterified CNCs.

\section{3.3. Structural and crystallinity changes of cellulose I}

272 The surface chemistry of transesterified CNCs was further characterized by Raman. The

273 spectra of unmodified CNC and CNCFE are shown in Fig. 3. The band of CNCFE at $1655 \mathrm{~cm}^{-1}$

274 was assigned to $\mathrm{C}=\mathrm{C}$ stretching of the hydrocarbon chain of $\mathrm{C} 18: 1, \mathrm{C} 18: 2$ and $\mathrm{C} 18: 3$. This is

275 consistent with the FTIR results. The weak ester $\mathrm{C}=\mathrm{O}$ stretching band appears at $1720 \mathrm{~cm}^{-1}$.

276 According to the method developed by Agarwal et al. (Agarwal, Ralph, et al., 2013; Agarwal,

277 Ralph, Reiner, \& Baez, 2015; Agarwal et al., 2010), FT-Raman spectroscopy was used to

278 characterize the crystalline structure of cellulose I. The extent of cellulose I crystallinity was

279 determined by the intensity ratio of the 380 and $1096 \mathrm{~cm}^{-1}$ bands from the Raman spectra, which 
was $43 \%$ and $47 \%$ for unmodified CNC and CNCFE samples, respectively. This crystallinity

281 was lower than values that were reported previously for CNCs, i.e., 54-66 \% (Agarwal, Reiner,

$282 \&$ Ralph, 2013; Chen et al., 2015), likely because there is a significant amount of cellulose II in

283 the CNCs produced from dissolving pulp obtained by alkaline pulping and acid hydrolysis

284 processes. Also, noteworthy is the fact that even for all cellulose I CNCs 380-Raman

285 crystallinities are usually significantly lower compared to XRD methods (Agarwal, Reiner, et al., 286 2013; Chen et al., 2015). Crystallinity of cellulose I was not significantly changed, suggesting its

287 crystal network wasn't interrupted by transesterification. Since the high degree of crystallinity of $288 \mathrm{CNC}$ is one of the major reasons given for its attractive mechanical, physical and chemical

289 properties (Habibi et al., 2010; Moon et al., 2013), it is expected that the mechanical strength is 290 maintained after modification with CME to form CNCFE.

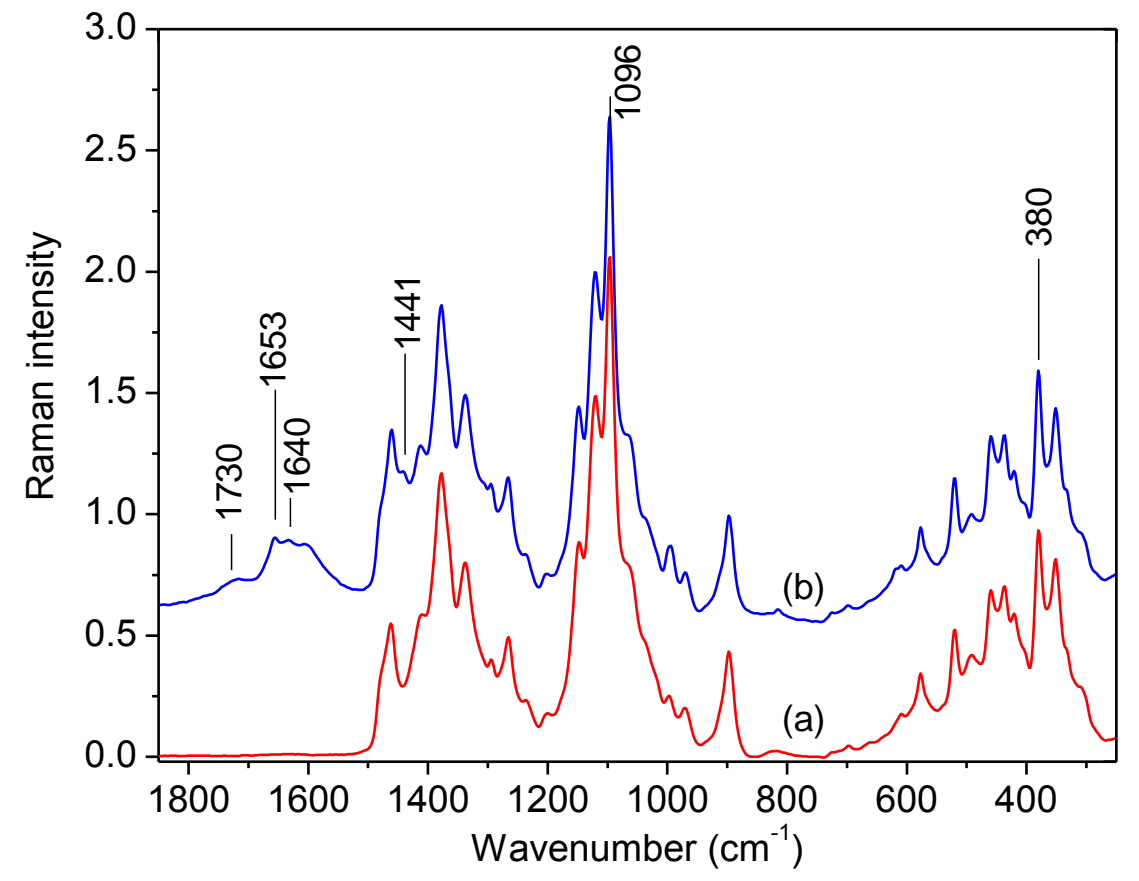

Fig. 3 Raman spectra in the region of $1850-250 \mathrm{~cm}^{-1}$ of (a) CNC and (b) CNCFE. 


\subsection{Thermal stability of nanocrystals}

The thermal stability of CNCFE was found to be higher than CME and unmodified CNCs as

293 shown in thermogravimetric (TG) and derivative thermogravimetric (DTG) curves (Fig. 4). The

294 mass loss due to thermal degradation of CME occurred in one stage with the onset degradation

295 temperature $\left(\mathrm{T}_{\text {onset }}\right)$ of $168^{\circ} \mathrm{C}$. During this stage CME was completely decomposed at $\mathrm{T}_{\text {com }}=$

$296307^{\circ} \mathrm{C}$. About $60 \mathrm{wt} \%$ of unmodified CNC sample was degraded at $\mathrm{T} \leq 350^{\circ} \mathrm{C}$ followed by the

297 second stage (350-450 $\mathrm{C}$ ) caused by the depolymerization and decomposition of cellulose

298 glycosyl units (Shen, Gu, \& Bridgwater, 2010; Wei, McDonald, \& Stark, 2015). The char

299 residual yield of unmodified $\mathrm{CNC}$ at $600^{\circ} \mathrm{C}$ was about $21 \mathrm{wt} \%$. The temperature of maximum

300 decomposition rate $\left(\mathrm{T}_{\max }\right)$ in $\mathrm{CNCFE}$ was $28^{\circ} \mathrm{C}$ higher than that of unmodified $\mathrm{CNC}\left(327^{\circ} \mathrm{C}\right)$,

301 while the residual at the end was about $16 \mathrm{wt} \%$. We conclude, therefore, that transesterification

302 improved the thermal stability of CNC with CME, even though CME itself is less thermally

303 stable. The improved thermal stability of transesterified nanocrystals could broaden the melt

304 processing window when being compounded with polymer for nanocomposites.

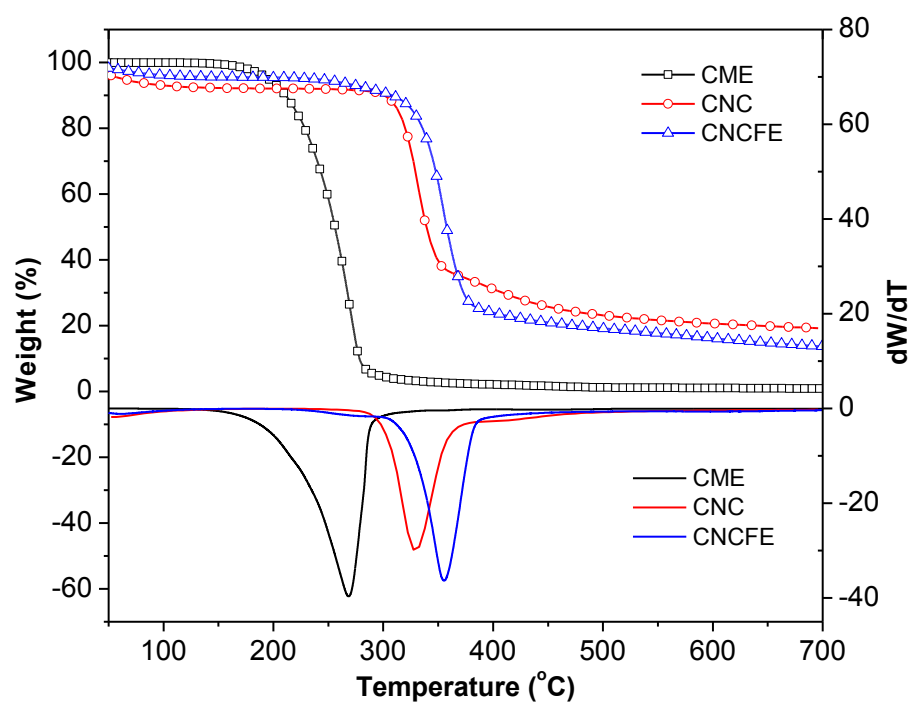

Fig. 4 TG and DTG curves of CME, CNC, and CNCFE. 
The TEM image in Fig. 5a shows the structure and dimension of CNCs produced from

307 dissolving pulp. It can be seen that CNCs have rod-like shape and were separated without

308 aggregation before the freeze-drying process. Size measurements were made using an image

309 analysis software Fiji (http://imagej.net/Fiji). The CNCs had a broad size distribution, with the

310 length and diameter ranges of $25-150 \mathrm{~nm}$ and 5-18 nm, respectively, and averages of 78 $\pm 30 \mathrm{~nm}$

311 and $11 \pm 2 \mathrm{~nm}$, respectively. The CNCs derived from sulfuric acid hydrolysis always have smaller

312 dimensions than those produced by other approaches (Chen et al., 2015; Klemm et al., 2011).

313 SEM micrographs of unmodified CNC and CNCFE surfaces are shown in Fig. 5b-5d. The

314 unmodified CNC suspension (10.7 wt\%) was directly deposited onto carbon tape and dried under

315 air before SEM examination. As shown in Fig. 5b, the air-dried CNCs are lamella/sheet like

316 flakes. The freeze-dried unmodified CNCs (Fig. 5c) lost the rod-like shape morphology as well.

317 The presence of micro-scale agglomerated CNCs with larger sizes than air-dried CNCs, as

318 shown in Fig. 5c, are possibly originated from the hydrogen-bonding and van der Waals forces

319 during freeze-drying of the CNC suspension (10.7 wt\%). Micro-scaled aggregates were found by

320 researches previously (Fumagalli, Sanchez, Boisseau, \& Heux, 2013; Han, Zhou, Wu, Liu, \&

$321 \mathrm{Wu}, 2013)$. Agglomerates sizes become smaller after transesterification. Moreover, the surface

322 structure of CNCFE agglomerated flakes seems to be rougher (Fig. 5d), which is likely caused

323 by the mechanically shearing/washing during the transesterification reaction. 

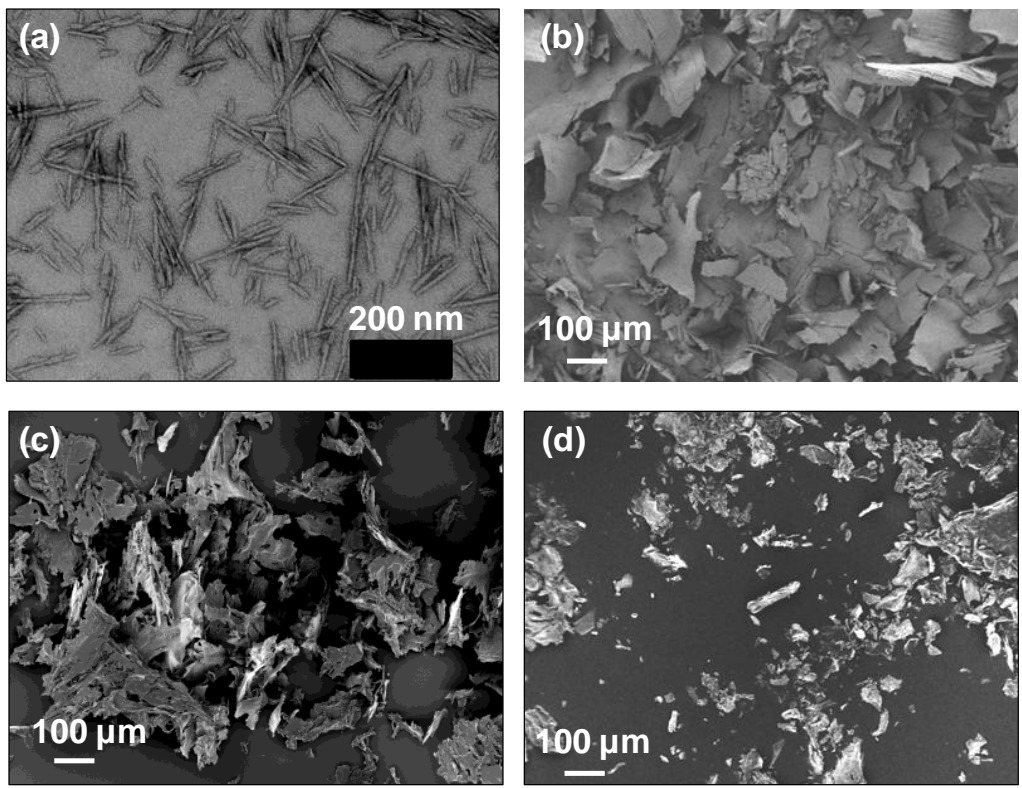

Fig. 5 Transmission electron micrograph of CNCs (a) and scanning electron micrographs of unmodified CNC suspension (10.7 wt\%) (b), freeze-dried unmodified CNCs (c) and CNCFE (d).

\subsection{Crystalline structure}

The effect of transesterification on the crystalline structure of unmodified CNC and CNCFE

327 was investigated by XRD analysis. The diffraction patterns are shown in Fig. 6. Unmodified

328 CNCs showed diffraction peaks at $14.5^{\circ}, 16.5^{\circ}, 22.3^{\circ}$, and $34.5^{\circ}$, being assigned to cellulose I

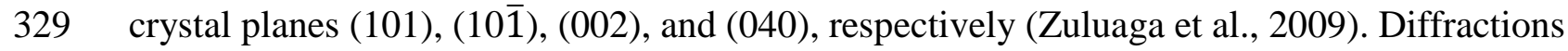

330 from cellulose II are also present in unmodified CNCs at $2 \theta$ of $12.5^{\circ}, 19.8^{\circ}(10 \overline{1}), 21.9^{\circ}$, and

$33134.5^{\circ}$. Cellulose I can be transformed to cellulose II during alkali pulping and acid hydrolysis

332 process originated from dissolving pulp preparation stage (El Oudiani, Chaabouni, Msahli, \&

333 Sakli, 2011). The ratio of peak intensity at $22.3^{\circ}$ vs. intensity at $19.8^{\circ}\left(\mathrm{I}_{22.3^{\circ}} \mathrm{I}_{19.8^{\circ}}\right)$ is used to

334 estimate the Cellulose I vs. cellulose II content (Gindl \& Keckes, 2005). The peak intensities

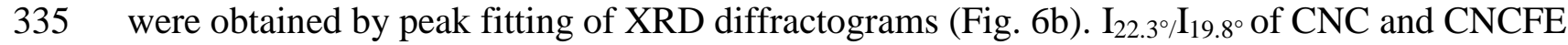

336 was 2.00 and 1.98, respectively, indicating the CNC crystalline structure was not influenced by 
337 this transesterification modification. Diffraction patterns of CNCFE are similar to those of

338 unmodified CNCs sample, suggesting transesterification did not change crystalline structure of

339 the nanocrystals. This was further supported by Raman spectroscopy (Fig. 3) where intensity of

340 bands that were associated with the crystalline structures was similar between unmodified CNCs

341 and CNCFE.

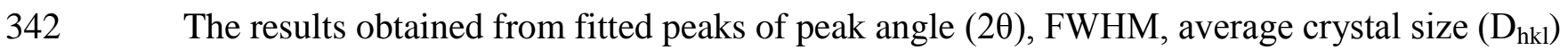

343 in the direction perpendicular to the reflection plane, and crystallinity indices ( $\left.\mathrm{CrI} \%_{\mathrm{CNC} / \mathrm{CNCFE}}\right)$

344 are listed in Table 2. The crystallinity indices were $76.9 \%$ for unmodified CNCs and $75.1 \%$ for

345 CNCFE, while the crystallinity of original wood pulp was about $76 \%$ (Chen et al., 2015). Hence,

346 crystallinity index of cellulose was not altered during transformation of wood pulp to CNCs. The

347 average crystal width for cellulose I and cellulose II were $44 \AA\left(D_{002}\right)$ and $69 \AA\left(D_{10} \overline{1}\right)$

348 respectively, for both unmodified and CNCFE. These values are consistent with reported values

349 of cellulose I and II nanocrystals (Sèbe, Ham-Pichavant, Ibarboure, Koffi, \& Tingaut, 2012). It is

350 therefore concluded that neither the crystal size nor the degree of crystallinity of CNCs were

351 affected by transesterification, and hence, crystal mechanical strength was expected to be

352 retained. This is consistent with the cellulose I crystallinity results from quantitative Raman

353 spectroscopic analysis discussed above. 

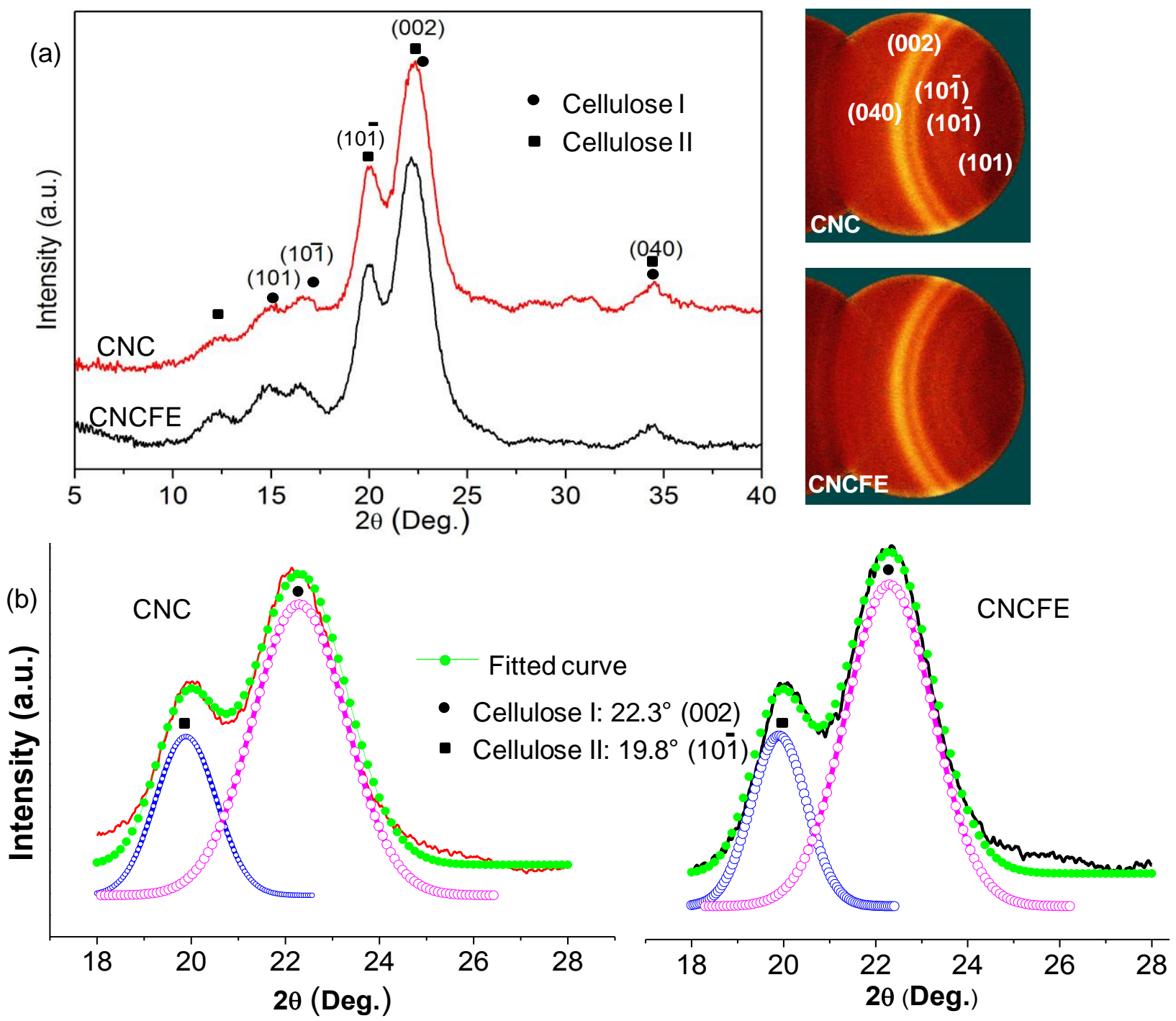

Fig. 6 XRD diffractograms and patterns (a) and fitted peaks for cellulose I and cellulose II (b) of unmodified CNC and CNCFE

Table 2. XRD results of unmodified CNC and $\mathrm{CNCFE}^{a}$.

\begin{tabular}{llllllll}
\hline & $2 \theta$ & FWHM $\left(^{\circ}\right)$ & $\mathrm{d}(\AA)$ & $\mathrm{D}_{002}(\AA)$ & $\mathrm{D}_{10} \overline{1}(\AA)$ & CrI\% $_{\text {CNC/CNCFE }}$ & Cell-I/Cell-II \\
\hline CNC & 20.0 & 1.165 & 8.9 & & 69 & & 1.98 \\
& 22.3 & 1.832 & 7.9 & 44 & & 76.9 & \\
CNCFE & 20.0 & 1.166 & 8.9 & & 69 & & \\
& 22.3 & 1.748 & 7.9 & 44 & & 75.1 & \\
\hline
\end{tabular}

${ }^{a}$ : Crystal sizes were determined in the direction perpendicular to the planes of (002) of cellulose

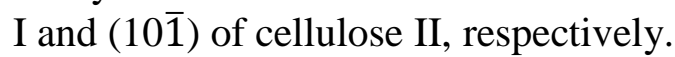




\section{$354 \quad 3.7$. Hydrophobicity changes}

355 The water contact angle results are given in Fig. 7. The dynamic contact angle changes with

356 time $(0-10 \mathrm{~s})$ showed that the CNCFE surface is significantly more hydrophobic, as indicated by

357 higher contact values of $\mathrm{CNCFE}\left(62^{\circ}\right)$ than the unmodified CNCs $\left(23^{\circ}\right)$ (Fig. 7a-7c). The

358 relatively lower contact angles were obtained as compared with acetylated CNC films as

359 reported previously, which could be contributed to the lower degree of $\mathrm{OH}$ substituted (Abraham

360 et al., 2016). The unmodified CNC surface showed water droplet spreading with time as

361 compared to the CNCFE. Photographs of suspensions of freeze-dried unmodified CNCs and the

362 CNCFE re-dispersed in water/hexane (Fig. 7d-7g). The suspension was treated with sonication

363 for 10 mins at $50 \%$ of maximum power using a tip-type Ultrasonic processor (Sonisc Vibracell,

364 ThermoFisher, USA), and then allowed for 30 mins before photographs were taken. The re-

365 dispersed freeze-dried unmodified CNCs formed a homogeneous and stable suspension in water

366 but settled in hexane, while the CNCFE settled in water but formed a stable suspension in hexane.

367 This confirmed further that the significantly increased hydrophobicity of tranesterified CNC

368 increased its dispersion ability in hexane, a non-polar solvent. 

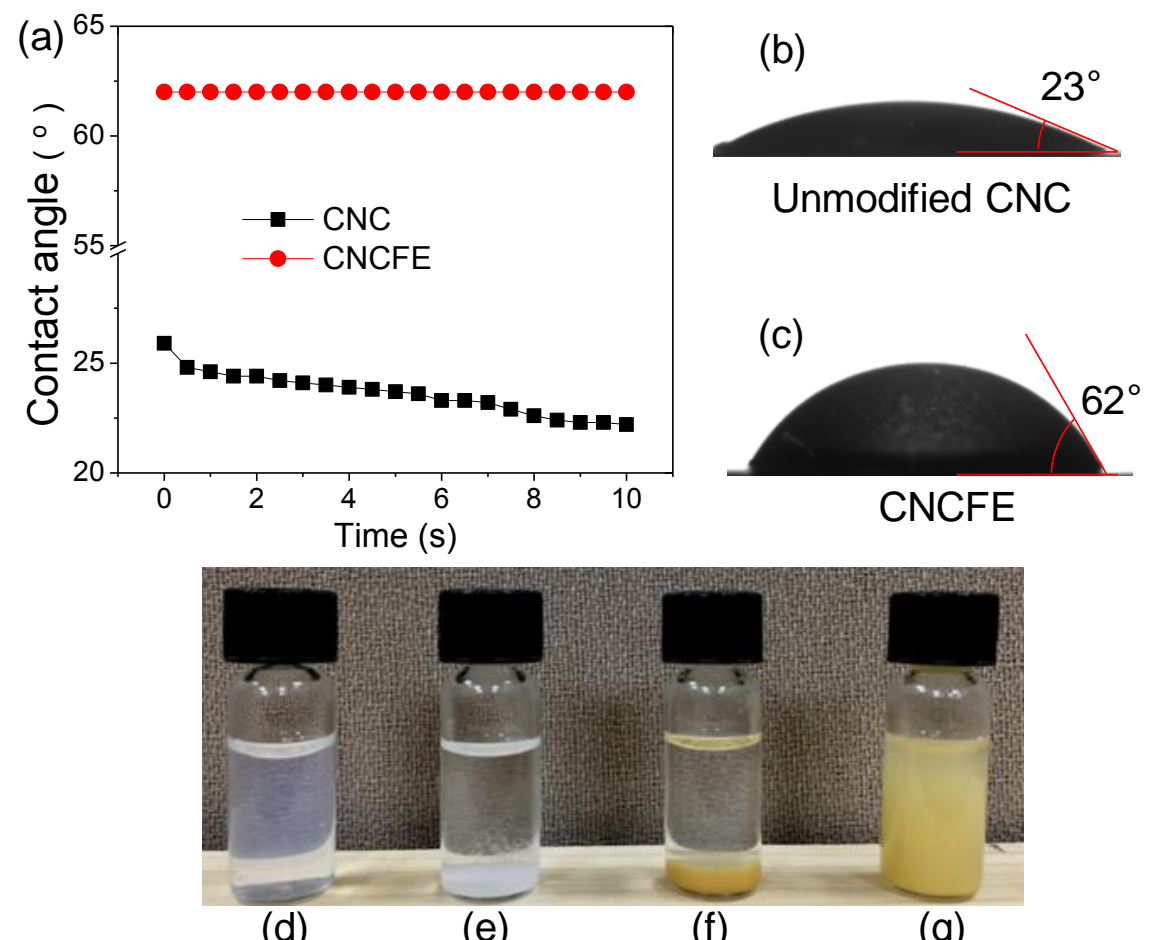

CNCFE

(d)

(e)

(f)

(g)

Fig. 7 Dynamic water contact angles versus time (a), water contact angles measured on surfaces of unmodified CNCs (b) and CNCFE (c) after 5s, and suspensions of unmodified CNCs in water (d) and hexane (e), and the CNCFE in water (f) and hexane (g).

\section{Conclusions}

Cellulose nanocrystals were successfully transesterified with a sustainable and green

372 chemistry-based approach using vegetable oil fatty acid methyl ester. This functionalization

373 method on cellulose nanomaterials is reported here for the first time. The degree of crystallinity

374 and crystalline structure of nanocrystals were not altered by functionalization. The transesterified

375 CNC showed improved thermal stability as compared to unmodified CNC. The particle sizes of

376 transesterified CNC agglomerates were smaller than unmodified equivalents. The transesterified

377 CNC surfaces are significantly more hydrophobic than the unmodified CNCs. This 
378 transesterification strategy has the potential to be employed to modify other cellulose

379 nanomaterials, i.e. $\mathrm{CNF}$, having higher availability of $\mathrm{OH}$ groups on surface. The byproduct,

380 crude glycerol, during the CME production process can be recycled and potentially used as

381 emulsions and polymer processing aids for future research. This research could broaden the

382 application of cellulose nanomaterials in the fields of coatings, emulsions, and nanocomposites,

383 and therefore facilitate its commercialization.

\section{Acknowledgement}

386 The authors thank the funding from Public-Private Partnership for Nanotechnology ( $\mathrm{P}^{3}$ Nano) US

387 Endowment for Forestry and Communities, Inc. (Grant No. CWFSCL15). We also acknowledge

388 the following people from the USDA Forest Service, Forest Products Laboratory: Richard S.

389 Reiner for preparing CNCs, Dr. Craig Clemons and Philip Walsh for help with TGA, Dr. J.Y.

390 Zhu and Huiyang Bian for water contact angle measurements, and Sally A. Ralph for Raman

391 experiments.

392

393 


\section{References}

395 Abraham, E., Kam, D., Nevo, Y., Slattegard, R., Rivkin, A., Lapidot, S., \& Shoseyov, O. (2016).

396 Highly Modified Cellulose Nanocrystals and Formation of Epoxy-Nanocrystalline Cellulose

397 (CNC) Nanocomposites. ACS Appl. Mater. Interfaces, 8(41), 28086-28095.

398 Agarwal, U. P., Ralph, S. A., \& Reiner, R. S. (2013). Estimation of Cellulose Crystallinity of 399 Lignocelluloses Using Near-IR FT-Raman Spectroscopy and Comparison of the Raman and 400 Segal-WAXS Methods. J. Agr. Food Chem., 61(1), 103-113.

401 Agarwal, U. P., Ralph, S. A., Reiner, R. S., \& Baez, C. (2015). Probing crystallinity of never402 dried wood cellulose with Raman spectroscopy. Cellulose, 23(1), 125-144.

403 Agarwal, U. P., Reiner, R. S., \& Ralph, S. A. (2010). Cellulose I crystallinity determination 404 using FT-Raman spectroscopy: univariate and multivariate methods. Cellulose, 17(4), 721-733.

405 Agarwal, U. P., Reiner, R. S., \& Ralph, S. A. (2013). Crystallinity of nanocellulose materials by 406 Near-IR FT-Raman spectroscopy. . In M. T. Postek, R. J. Moon, A. W. Rudie \& M. A. Bilodeau 407 (Eds.), Production and Applications of Cellulose Nanomaterials (pp. 43-44). Peachtree Corners, 408 GA: TAPPI Press

409 Alexander, L. E. (1969). X-ray Diffraction Method in Polymer Science. New York, USA Wiley410 Interscience.

411 Bardet, R., Roussel, F., Coindeau, S., Belgacem, N., \& Bras, J. (2015). Engineered pigments 412 based on iridescent cellulose nanocrystal films. Carbohydr. polym., 122, 367-375.

413 Boujemaoui, A., Mongkhontreerat, S., Malmström, E., \& Carlmark, A. (2015). Preparation and 414 characterization of functionalized cellulose nanocrystals. Carbohydr. polym., 115, 457-464. 
415 Chen, L., Wang, Q., Hirth, K., Baez, C., Agarwal, U. P., \& Zhu, J. Y. (2015). Tailoring the yield 416 and characteristics of wood cellulose nanocrystals (CNC) using concentrated acid hydrolysis. 417 Cellulose, 22(3), 1753-1762.

418 Chen, L., Zhu, J. Y., Baez, C., Kitin, P., \& Elder, T. (2016). Highly thermal-stable and functional 419 cellulose nanocrystals and nanofibrils produced using fully recyclable organic acids. Green 420 Chem., 18(13), 3835-3843.

421 El Oudiani, A., Chaabouni, Y., Msahli, S., \& Sakli, F. (2011). Crystal transition from cellulose I 422 to cellulose II in $\mathrm{NaOH}$ treated Agave americana L. fibre. Carbohydr. polym., 86(3), 1221-1229.

423 Espino-Pérez, E., Domenek, S., Belgacem, N., Sillard, C., \& Bras, J. (2014). Green Process for 424 Chemical Functionalization of Nanocellulose with Carboxylic Acids. Biomacromolecules, 425 15(12), 4551-4560.

426 Eyley, S., \& Thielemans, W. (2011). Imidazolium grafted cellulose nanocrystals for ion 427 exchange applications. Chem. Commun., 47(14), 4177.

428 Fumagalli, M., Sanchez, F., Boisseau, S. M., \& Heux, L. (2013). Gas-phase esterification of 429 cellulose nanocrystal aerogels for colloidal dispersion in apolar solvents. Soft Matter, 9(47), $430 \quad 11309-11317$.

431 Gindl, W., \& Keckes, J. (2005). All-cellulose nanocomposite. Polymer, 46(23), 10221-10225.

432 Habibi, Y., Lucia, L. A., \& Rojas, O. J. (2010). Cellulose nanocrystals: Chemistry, self-assembly, 433 and applications. Chem. Rev., 110, 3479-3500.

434 Han, J., Zhou, C., Wu, Y., Liu, F., \& Wu, Q. (2013). Self-Assembling Behavior of Cellulose 435 Nanoparticles during Freeze-Drying: Effect of Suspension Concentration, Particle Size, Crystal 436 Structure, and Surface Charge. Biomacromolecules, 14(5), 1529-1540. 
437 Hatton, F. L., Kedzior, S. A., Cranston, E. D., \& Carlmark, A. (2017). Grafting-from cellulose 438 nanocrystals via photoinduced $\mathrm{Cu}$-mediated reversible-deactivation radical polymerization.

439 Carbohydr. polym., 157, 1033-1040.

440 He, B. B., Singh, A. P., \& Thompson, J. C. (2005). Experimental optimization of a continuous-

441 flow reactive distillation reactor for biodiesel production. Transactions of the ASAE, 48(6), 2237-

4422243.

443 Huang, S., Wang, X., Shen, J., Wu, R., Zhao, H., Wang, Y., . . X Xia, Y. (2016). Surface

444 functionalization of cellulose nanocrystals with polymeric ionic liquids during phase transfer.

445 Carbohydr. polym.

446 Jabbari, E., Wisniewski, N., \& Peppas, N. A. (1993). Evidence of mucoadhesion by chain

447 interpenetration at a poly (acrylic acid)/mucin interface using ATR-FTIR spectroscopy. $J$.

448 Control. Release, 26(2), 99-108.

449 Klemm, D., Heublein, B., Fink, H.-P., \& Bohn, A. (2005). Cellulose: Fascinating Biopolymer

450 and Sustainable Raw Material. Angew. Chem. Int. Ed., 44(22), 3358-3393.

451 Klemm, D., Kramer, F., Moritz, S., Lindström, T., Ankerfors, M., Gray, D., \& Dorris, A. (2011).

452 Nanocelluloses: A New Family of Nature-Based Materials. Angew. Chem. Int. Ed., 50(24), 5438-

4535466.

454 Lang, X., Dalai, A. K., Bakhshi, N. N., Reaney, M. J., \& Hertz, P. B. (2001). Preparation and 455 characterization of bio-diesels from various bio-oils. Bioresource Technol., 80(1), 53-62.

456 Lin, N., Huang, J., Chang, P. R., Feng, J., \& Yu, J. (2011). Surface acetylation of cellulose 457 nanocrystal and its reinforcing function in poly(lactic acid). Carbohydr. polym., 83(4), 18344581842. 
Mayo, D. W., Miller, F. A., \& Hannah, R. W. (2003). Course Notes on The Interpretation of

460 Infrared and Raman Spectra Hoboken, New Jersey: John Wiley \& Sons, Inc.

461 Moon, R. J., Beck, S., \& Rudie, A. W. (2013). Cellulose Nanocrystals - A Material with Unique

462 Properties and Many Potential Applications In M. T. Postek, R. J. Moon, A. W. Rudie \& M. A.

463 Bilodeau (Eds.), Production and Applications of Cellulose Nanomaterials (pp. 9-12). Peachtree

464 Corners, GA: TAPPI Press

465 Morandi, G., Heath, L., \& Thielemans, W. (2009). Cellulose Nanocrystals Grafted with

466 Polystyrene Chains through Surface-Initiated Atom Transfer Radical Polymerization (SI-ATRP).

467 Langmuir, 25(14), 8280-8286.

468 Pereira, A. L. S., Nascimento, D. M. d., Souza Filho, M. d. s. M., Morais, J. P. S., Vasconcelos,

469 N. F., Feitosa, J. P. A., . . Rosa, M. d. F. (2014). Improvement of polyvinyl alcohol properties

470 by adding nanocrystalline cellulose isolated from banana pseudostems. Carbohydr. polym., 112,

$471 \quad 165-172$.

472 Ramírez, J. A. Á., Fortunati, E., Kenny, J. M., Torre, L., \& Foresti, M. L. (2016). Simple citric

473 acid-catalyzed surface esterification of cellulose nanocrystals. Carbohydr. polym.

474 Reiner, R., \& Rudie, A. (2013). Process Scale-Up of Cellulose Nanocrystal Production to $25 \mathrm{~kg}$

475 per Batch at the Forest Products Laboratory In M. T. Postek, R. J. Moon, A. W. Rudie \& M. A.

476 Bilodeau (Eds.), Production and Applications of Cellulose Nanomaterials (pp. 21-24). Peachtree

477 Corners, GA: TAPPI Press

478 Sèbe, G., Ham-Pichavant, F., Ibarboure, E., Koffi, A. L. C., \& Tingaut, P. (2012).

479 Supramolecular Structure Characterization of Cellulose II Nanowhiskers Produced by Acid

480 Hydrolysis of Cellulose I Substrates. Biomacromolecules, 13(2), 570-578. 
481 Segal, L., Creely, J., Martin Jr., A., \& Conrad, C. (1959). An empirical method for estimating the 482 degree of crystallinity of native cellulose using the X-ray diffractometer. Text. Res. J., 29(10), $483 \quad 786-794$.

484 Shen, D. K., Gu, S., \& Bridgwater, A. V. (2010). The thermal performance of the 485 polysaccharides extracted from hardwood: Cellulose and hemicellulose. Carbohydr. polym., $486 \quad 82(1), 39-45$.

487 Wang, Q. Q., Zhu, J. Y., Reiner, R. S., Verrill, S. P., Baxa, U., \& McNeil, S. E. (2012). 488 Approaching zero cellulose loss in cellulose nanocrystal (CNC) production: recovery and 489 characterization of cellulosic solid residues (CSR) and CNC. Cellulose, 19(6), 2033-2047.

490 Wei, L., \& McDonald, A. (2016). A Review on Grafting of Biofibers for Biocomposites 491 Materials, 9(4), 303, doi: 310.3390/ma9040303.

492 Wei, L., McDonald, A. G., Freitag, C., \& Morrell, J. J. (2013). Effects of wood fiber 493 esterification on properties, weatherability and biodurability of wood plastic composites. Polym. 494 Degrad. Stab., 98(7), 1348-1361.

495 Wei, L., McDonald, A. G., \& Stark, N. M. (2015). Grafting of Bacterial Polyhydroxybutyrate 496 (PHB) onto Cellulose via In Situ Reactive Extrusion with Dicumyl Peroxide. Biomacromolecules, 497 16(3), 1040-1049.

498 Yoo, Y., \& Youngblood, J. P. (2016). Green One-Pot Synthesis of Surface Hydrophobized 499 Cellulose Nanocrystals in Aqueous Medium. ACS Sustainable Chem. Eng., 4(7), 3927-3938.

500 Zaman, M., Xiao, H., Chibante, F., \& Ni, Y. (2012). Synthesis and characterization of 501 cationically modified nanocrystalline cellulose. Carbohydr. polym., 89(1), 163-170. 
502 Zuluaga, R., Putaux, J. L., Cruz, J., Vélez, J., Mondragon, I., \& Gañán, P. (2009). Cellulose 503 microfibrils from banana rachis: Effect of alkaline treatments on structural and morphological 504 features. Carbohydr. polym., 76(1), 51-59. 